\title{
The Generalized State-Space Description of Positive Realness and Bounded Realness ${ }^{1}$
}

\author{
He-Sheng Wang and Fan-Ren Chang \\ Department of Electrical Engineering \\ National Taiwan University \\ Taipei, Taiwan 10617 \\ Republic of China
}

\begin{abstract}
Positive realness and bounded realness are two important issues in system theory. Some related applications about these notions include analysis and synthesis of passive networks, optimal designs in control and estimation, stability investigations in linear/nonlinear closed-loops; etc.. In this paper, we propose the equations of constant matrices, which are stabilizable and detectable realizations of impulse-free generalized state-space systems, to describe the positive real and bounded real properties. The established generalized positive real lemma and generalized bounded real lemma are necessary and sufficient. They are the extensions of results in [5], in which the statespace realizations are considered. The impulse-free generalized state-space systems contain both finite and nondynamic infinite modes. The state-space systems contain finite modes only. To express those algebraic restrictions among state variables is easier in the generalized state-space.
\end{abstract}

\section{Introduction}

The studies of positive realness and bounded realness have played essential roles in linear time-invariant lumped passive network analysis and synthesis. There are two important lemmas to interpret the positive real and bounded real constraints in terms of the matrices of a state-space realization of the given transfer function [5]. The applications of the positive real lemma include the followings: spectral factorization in robust control [10], [17] and Wiener-Hopf design [12], [24], stability analysis in circle criterion [5] and Popov criterion [5], [22]; etc.. Also, the applications of the bounded real lemma are $H_{\infty}$ control [10], innerouter factorization [1], [10] for model reduction; etc..

The state-space representation of a dynamic system has been extended to the generalized statespace representation recently [9], [15], [21]. The behaviors of a generalized state-space system (also called as a singular system or a descriptor system)

\footnotetext{
${ }^{1}$ This work is supported by the National Council of the Repub-
} lic of China under Grant NSC 85-2213-E-002-003 contain exponential modes, nondynamic modes and impulsive modes. The another terms of these three conducts are finite poles, static infinite poles and dynamic infinite poles respectively. The state-space systems possess exponential modes only, however. Also, the algebraic restriction between state variables can be explicitly expressed by the generalized state-space representation. To describe those restricted state variables in state-space representation is difficult in general.

In this paper, we will extended the interpretation for the positive real and the bounded real properties from the state-space [5] to the generalized state-space. In section 2 , the definitions of positive real and bounded real in terms of frequency domain are briefly reviewed. Then, some results about linear-quadratic optimal regulation problems for singular systems are summarized therein. The generalized algebraic Riccati equations are needed in the next section. The generalized positive real lemma and the generalized bounded real lemma are proposed in section 3. The proposed lemmas are established on the stabilizable and detectable impulse-free generalized state-space systems. Our results are sufficient and necessary. The conclusions are finally given in section 4 .

\section{Preliminaries}

First, we give the definitions about positive real and bounded real systems. There are several different definitions of a positive real system. One can see [5], [6]: [10], [18]. Throughout this paper, we will use the following definition [18].

Definition 1: Consider a transfer function matrix $G(s)$, and assume that $G(s)$ is square. Then

1. $G(s)$ is said to be positive real (PR) if $G(s)$ is analytic in $\operatorname{Re}(s)>0$ and $G(s)+G^{\prime}\left(s^{*}\right) \geq 0, \forall$ $\operatorname{Re}(s)>0$.

2.G(s) is said to be strictly positive real (SPR) if it is . analytic in $\operatorname{Re}(s) \geq 0$ and satisfies $G(j \omega)+\left(\mathcal{J}^{\prime}(-j \omega)>0, \forall \omega \in[0, \infty)\right.$.

3. $G(s)$ is said to be extended strictly positive real (ESPR) if it is SPR and $G(j \infty)+G^{\prime}(-j \infty)>0$. 
The above definitions are expressed in the frequency domain. However, the time domain property of positive realness can be viewed as follows.

Definition 2[5]: A system $\Sigma$ (see Fig. 1) is said to be positive real if for all real-valued piecewise continuous function $u(\bullet)$, and $t \geq 0$

$$
0 \leq \int_{0}^{t} y^{\prime}(\tau) u(\tau) d \tau
$$

whenever the system is relaxed at time $t=0$. (i.e. initial state is zero)

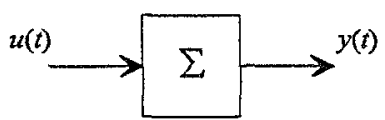

Fig. 1 System $\Sigma$, its input $u(t)$ and output $y(t)$

where $y(\bullet), u(\bullet) \in R^{n \times 1}$

The definition of bounded realness is fairly standard, we will use the following definition throughout this paper.

Definition 3[10]: Consider an $m \times m$ matrix of real rational functions $S(s)$. $S(s)$ is bounded real if it is analytic in $\operatorname{Re}(s) \geq 0$ and $I-S^{\prime}(-j \omega) S(j \omega) \geq 0$

for all real $\omega$. Furthermore, $S(s)$ is strictly bounded real if it is bounded real and $I-S^{\prime}(-j \omega) S(j \omega)>0$.

We will review some results on the linearquadratic optimal regulation problem for generalized state-space systems, which will be useful for the proofs of the next section, in the remaining part of this section.

Consider the continuous-time linear timeinvariant generalized state-space system $\Sigma$

$$
\begin{aligned}
E \dot{x}(t) & =A x(t)+B u(t) \\
y(t) & =C x(t)+D u(t), \quad E x(0-)=E x_{0}
\end{aligned}
$$

where $E \in R^{n \times n}, A \in R^{n \times n}, B \in R^{n \times m}, C \in R^{p \times m}$ and $D \in R^{p \times n}, x(t)$ is the $\mathrm{n}$-dimensional state vector, $u(t)$ is the $m$ dimensional control vector and $y(t)$ is the $p$ dimensional output vector. The matrix $E$ is, in general, a singular matrix with $\operatorname{rank} E=r<n$.

If there exists a state-variable feedback $u(t)=K x(t)$, such that the feedback system $E \dot{x}(t)=(A+B K) x(t)$ is regular, impulse-free and stable, then $\{E, A+B K\}$ is said to be admissible and the feedback gain $\mathrm{K}$ is called an admissible feedback gain [7], [13].

Now, consider a linear quadratic regulation problem with the cost function

$$
J\left(E x_{0}, u\right)=\frac{1}{2} \int_{0}^{T}\left[\begin{array}{ll}
x^{\prime}(t) & u^{\prime}(t)
\end{array}\right]\left[\begin{array}{cc}
Q & S \\
S^{\prime} & R
\end{array}\right]\left[\begin{array}{l}
x(t) \\
u(t)
\end{array}\right] d t
$$

where $Q$ is nonnegative definite, $R$ is positive definite. Choose the Hamiltonian function $H(x, \lambda, u)$ as

$$
H(x, \lambda, u)=\frac{1}{2}\left[\begin{array}{ll}
x^{\prime} & u^{\prime}
\end{array}\right]\left[\begin{array}{cc}
Q & S \\
S^{\prime} & R
\end{array}\right]\left[\begin{array}{l}
x \\
u
\end{array}\right]+\lambda^{\prime}(A x+B u) .
$$

For solving the optimization problem, the following assumption is made

Assumption 1: The generalized state-space system (1), (2) is regular, impulse-free and finite dynamics stabilizable [7], [13], [15].

According to the theory of the Lagrange multiplier, we know that the necessary conditions for $J$ to be minimal is that

$$
E \dot{x}=\frac{\partial H}{\partial \lambda}, E^{\prime} \dot{\lambda}=-\frac{\partial H}{\partial x}, \frac{\partial H}{\partial u}=0,
$$

which will result in the following homogeneous singular system (or called the Hamiltonian system)

$$
\left[\begin{array}{ccc}
E & 0 & 0 \\
0 & E^{\prime} & 0 \\
0 & 0 & 0
\end{array}\right]\left[\begin{array}{l}
\dot{x} \\
\dot{\lambda} \\
\dot{u}
\end{array}\right]=\left[\begin{array}{ccc}
A & 0 & B \\
-Q & -A^{\prime} & -S \\
S^{\prime} & B^{\prime} & R
\end{array}\right]\left[\begin{array}{l}
x \\
\lambda \\
u
\end{array}\right]
$$

The following assumption is made for the system (3)

Assumption 2: The system (3) is regular impulsefree and no finite dynamic modes lie on the imaginary axis.

For the detail solution of this regulation problem, one can see [12], [13]. In this paper, we will need the following result of the infinitehorizon problem (i.e. taking limit of $T \rightarrow \infty$ ).

Lemma 1[13]: Under Assumptions 1 and 2, there exists a solution $X \in R^{n \times n}$ satisfying the following generalized algebraic Riccati equations (GARE)

$$
\begin{gathered}
X^{\prime} A+A^{\prime} X+Q-\left(X^{\prime} B+S\right) R^{-1}\left(S^{\prime}+B^{\prime} X\right)=0 \\
X^{\prime} E=E^{\prime} X
\end{gathered}
$$

such that $\{E, A+B K\}$ is admissible, where $K^{\Delta}=-R^{-1}\left(S^{\prime}+B^{\prime} X\right)$.

\section{Main Results}

In this section, we will derive the generalized positive real lemma and the generalized bounded real lemmas. Sufficient and necessary conditions for an impulse-free generalized state-space system to be positive real or bounded real are proposed. 
Let $G(s)=D+C(s E-A)^{-1} B$. Throughout this section, we will further assume $p=m$, i.e. $G(s)$ is square.

Assume that $\{E, A\}$ is regular and impulsefree, $\{E, A, B\}$ is finite dynamics stabilizable and $\{E, A, C\}$ is finite dynamics detectable [8], [7] [13].

The following lemmas are needed

\section{Lemma 2[5]: Consider the cost function}

$$
V\left(x_{0}, u, t\right)=\int_{0}^{t_{1}}\left(u^{\prime} R u+2 x^{\prime} C^{\prime} u\right) d t
$$

subject to the system $\Sigma$, where $R=D^{\prime}+D$ is positive definite. Then, there exists a lower bound for the cost function if and only if the system $\Sigma$ is positive real.

Remark: The cost function can be rewritten as

$$
V\left(x_{0}, u, t\right)=\int_{0}^{t_{1}}\left(y^{\prime}(t) u(t)+u^{\prime}(t) y(t)\right) d t=2 \int_{0}^{t_{1}}\left(y^{\prime}(t) u(t)\right) d t
$$

If $u(t) \equiv 0$, then $V \equiv 0$ for all $x_{0}$ and $t_{1}$. We see that the optimal solution for the cost function $V$ must, at least, be less than zero.

Lemma 3[20]: (Generalized Lyapunov Theorem) Let $\{E, A\}$ be regular and $\{E, A, C\}$ be impulse observable and finite dynamics detectable. Then $\{E, A\}$ is stable and impulsefree if and only if there exists a solution $X$ to the generalized Lyapunov equation (GLE)

$$
A^{\prime} X+X^{\prime} A+C^{\prime} C=0, E^{\prime} X=X^{\prime} E \geq 0 .
$$

We are now ready to show the generalized positive real lemma and the generalized bounded real lemma.

\section{Lemma 4: Generalized Positive Real Lemma}

Consider the system $\Sigma$. Assume that $R=D+D^{\prime}>0$ and no finite dynamic modes of $\{E, A\}$ lie on the imaginary axis. Then $G(s)$ is ESPR if and only if there exist matrices $P, L$ and $W_{0}$ with the appropriate dimensions, such that

$$
\begin{aligned}
& A^{\prime} P+P^{\prime} A=-L^{\prime} L, \\
& P^{\prime} B=C^{\prime}-L^{\prime} W_{0}, \\
& W_{0}^{\prime} W_{0}=D+D^{\prime}, \\
& E^{\prime} P=P^{\prime} E \geq 0 .
\end{aligned}
$$

Proof.
Sufficiency: Equations (4a) and (4d) satisfy the generalized Lyiapunov theorem, guarantee that $\{E$, $A\}$ is stable. So, $G(s)$ is analytic.in the right half plane. Next, we must check the nonnegativity of $G^{*}(s)+G(s)$.

$$
\begin{aligned}
& G^{*}(s)+G(s) \\
= & D^{\prime}+D+B^{\prime}\left(s^{*} E^{\prime}-A^{\prime}\right)^{-1} C^{\prime}+C(s E-A)^{-1} B \\
= & \left.W_{0}^{\prime} W_{0}+B^{\prime} \mid\left(s^{*} E^{\prime}-A^{\prime}\right)^{-1} P^{\prime}+P(s E-A)^{-1}\right] B+ \\
& B^{\prime}\left(s^{*} E^{\prime}-A^{\prime}\right)^{-1} L^{\prime} W_{0}+W_{0}^{\prime} L(s E-A)^{-1} B \\
= & W_{0}^{\prime} W_{0}+B^{\prime}\left(s^{*} E^{\prime}-A^{\prime}\right)^{-1}\left[P^{\prime} E\left(s+s^{*}\right)-P^{\prime} A-A^{\prime} P\right] \mathrm{x} \\
& (s E-A)^{-1} B+B^{\prime}\left(s^{*} E^{\prime}-A^{\prime}\right)^{-1} L^{\prime} W_{0}+W_{0}^{\prime} L(s E-A)^{-1} B \\
= & W_{0}^{\prime} W_{0}+B^{\prime}\left(s^{*} E^{\prime}-A^{\prime}\right)^{-1} L^{\prime} L(s E-A)^{-1} B \\
+ & B^{\prime}\left(s^{*} E^{\prime}-A^{\prime}\right)^{-1} L^{\prime} W_{0} \\
+ & W_{0}^{\prime} L(s E-A)^{-1} B+B^{\prime}\left(s^{*} E^{\prime}-A^{\prime}\right)^{-1} P^{\prime} E(s E-A)^{-1} B\left(s+s^{*}\right) \\
= & {\left[W_{0}^{\prime}+B^{\prime}\left(s^{*} E^{\prime}-A^{\prime}\right)^{-1} L\right]\left[W_{0}+L(s E-A)^{-1} B\right]+} \\
& B^{\prime}\left(s^{*} E^{\prime}-A^{\prime}\right)^{-1} P^{\prime} E(s E-A)^{-1} B\left(s+s^{*}\right)
\end{aligned}
$$

Since $P^{\prime} E=E^{\prime} P \geq 0$ and $\mathrm{W}_{0}{ }^{\prime} \mathrm{W}_{0}$ form the last equation, we see that: $G^{*}(s)+G(s)>0$ whenever $\operatorname{Re}(s) \geq 0$.

Necessity: From Lemma 2 and the remark therein, we see that there exist a optimal solution to the cost function

$$
V=\int_{0}^{t_{1}}\left(u^{\prime} R u+2 x^{\prime} C^{\prime} u\right) d t .
$$

By solving the optimization problem, we will meet the following Hamiltonian system

$$
\underbrace{\left[\begin{array}{ccc}
E & 0 & 0 \\
0 & E^{\prime} & 0 \\
0 & 0 & 0
\end{array}\right]\left[\begin{array}{c}
\dot{x} \\
\dot{\lambda} \\
\dot{u}
\end{array}\right]}_{\mathcal{E}}=\underbrace{\left[\begin{array}{ccc}
A & 0 & B \\
0 & -A^{\prime} & C^{\prime} \\
C & B^{\prime} & R
\end{array}\right]}_{\mathscr{H}}\left[\begin{array}{l}
x \\
\lambda \\
u
\end{array}\right]
$$

$\operatorname{det}\left(s \varepsilon-\Upsilon^{\prime}\right)=\operatorname{det}\left(s E^{\prime}-A\right) \operatorname{det}\left(s E^{\prime}+A^{\prime}\right) \operatorname{det}\left[G^{\prime}(-s)+G(s)\right]$

since $\{E, A\}$ has no pure imaginary finite-dynamic modes and $G(s)$ is $\operatorname{ESPR},\{\varepsilon, \varkappa\}$ will not have finite-dynamic modes lying on the imaginary axis. Therefore, Lemma 1 also guarantees there exists an optimal solution: to the cost function. Also, from Lemma 1, the solution $\mathrm{X}$ will satisfy the following GARE

$$
\begin{gathered}
X^{\prime} A+A^{\prime} X-\left(X^{\prime} B+C\right) R^{-1}\left(X^{\prime} B+C^{\prime}\right)^{\prime}=0 \\
E^{\prime} X=X^{\prime} E \leq 0
\end{gathered}
$$

choose

$$
P=-X, \quad W_{0}=R^{1 / 2}, \quad L=R^{-1 / 2}\left(B^{\prime} X+C\right),
$$

will meet the matrix equation (4a) (4d). Q.E.D',

Lemma 5: Generalized Bounded Real Lemma Consider the system $\Sigma$. Assume that $R=I-D^{\prime} D>0$ and no finite dynamic modes of 
$\{E, A\}$ lie on the imaginary axis. Then $\mathrm{G}(\mathrm{s})$ is strictly bounded real if and only if there exist matrices $P, L$ and $W_{0}$ with the appropriate dimensions, such that

$$
\begin{aligned}
& A^{\prime} P+P^{\prime} A=-C^{\prime} C-L^{\prime} L, \\
& -P^{\prime} B=C^{\prime} D+L^{\prime} W_{0}, \\
& I-D^{\prime} D=W_{0}^{\prime} W_{0}, \\
& E^{\prime} P=P^{\prime} E \geq 0
\end{aligned}
$$

Proof. The proof can be made in a similar manner as in [5].

\section{Conclusions}

We have developed the generalized positive real lemma and the generalized bounded real lemma. The results are all necessary and sufficient. State-space representations can be considered as special cases in generalized state-space representations whose matrices $E$ are nonsingular. Hence, our derivations will cover a wider range. However, there are still confined conditions: dynamic infinite modes and pure imaginary finite modes are not allowed in the proposed lemmas. How to relax these limitations will leave as future works.

\section{Reference}

[1] B.D.O. Anderson, "Algebraic Description of Bounded Real Matrices," Electronics Letters, vol. 2, No. 12, pp. 464-465, 1966.

[2] B.D.O. Anderson, "An Algebraic Solution to the Spectral Factorization Problem," IEEE Trans. on Automatic Control, vol. AC-12, No. 4, pp. 410-414, 1967.

[3] B.D.O. Anderson and J.B. Moore, "Algebraic Structure of Generalized Positive Real Matrices," SIAM J. Control, vol. 6, No. 4, pp. 615-624, 1968.

[4] B.D.O. Anderson and J.B. Moore, Optimal Control - Linear Quadratic Methods, Prentice-Hall, Englewood Cliffs, N.J., 1990.

[5] B.D.O. Anderson and S. Vongpanitlerd, Network Analysis and Synthesis - A Modern Systems Theory Approach, Prentice-Hall, Englewood Cliffs, N.J., 1973.

[6] N. Balabanian and T.A. Bickart, Electrical Network Theory, John Wiley \& Sons, New York, 1969.

[7] J. Bender and A.J. Laub, "The Linear-Quadratic Regulator for Descriptor Systems," IEEE Trans. on Automatic Control, vol. AC-23, No. 1, pp. 672-688, 1987.

[8] D. Cobb, "Controllability, Observability, and Duality in Singular Systems," IEEE Trans. on Automatic Control, vol. AC-29, No. 12, pp. 1076-1082, 1984.
[9] L. Dai, Singular Control Systems, (Lecture Notes in Control and Information Sciences, vol. 118) SpringerVerlag, New York, 1989.

[10] M. Green and D.J.N. Limebeer, Linear Robust Control, Prentice-Hall, Englewood Cliffs, N.J., 1995.

[11] F.R. Gantmacher, The Theory of Matrices, Chelsea, New York, 1959.

[12] I. Gohberg and M.A. Kaashoek, Constructive Methods of Wiener-Hopf Factorization, Birkhauser Verlag Basel, Boston, 1986.

[13] T. Katayama and K. Minamino, "Linear Quadratic Regulator and Spectral Factorization for ContinuousTime Descriptor Systems," Proceeding of 31th Conference on Decision and Control, Tucson, Arizona, December, pp. 967-972,1992.

[14] T. Katayama, K. Minamino and N. Morihira, "On the J-Spectral Factorization Using Descriptor Transfer Matrices," Proceedings of the 32nd Conference on Decision and Control, San Antonio, Texas, December, pp. 3304-3305, 1993.

[15] F.L. Lewis, "A Survey of Linear Singular Systems," Circuits Syst. Signal Process., vol. 5, No. 1, pp. 3-36. 1986.

[16] F.L. Lewis, Optimal Control, John Wiley \& Sons, New York, 1986.

[17] A.A. Stoorvogel, The Hoo Control Problem: A State-Space Approach, Prentice-Hall, Englewood Cliffs, N.J.; 1992.

[18] W. Sun, P.P. Khargonekar and D. Shim, "Solution to the Positive Real Control Problem for Linear Time-Invariant Systems," IEEE Trans. on Automatic Control, vol. AC-39, N0. 10, pp. 2034-2046, 1994.

[19] K. Takaba, N. Morihira and T. Katayama, "Hळ Control for Descriptor Systems- A J-Spectral Factorization Approach," Proceeding of 33rd Conference on Decision and Control, Lake Buena Vista, FL, December, pp. 2251-2256, 1994.

[20] K. Takaba, N. Morihira, and T. Katayama, "A Generalized Lyapunov Theorem for Descriptor System," Systems \& Control Letters, vol. 24, pp. 49-51, 1995.

[21] G. Verghese, B.C. Levy and T: Kailath, "A Generalized State-Space for Singular Systems," IEEE Trans. on Automatic Control, vol. AC-26, No. 4, pp. 811-831, 1981.

[22] M. Vidyasagar, Nonlinear Systems Analysis, 2nd edition, Prentice-Hall, Englewood Cliffs, N.J., 1993..

[23] D.C. Youla, "On the Factorization of Rational Matrices," IRE Trans. on Information Theory, vol. IT-7, No. 3, pp. 172-189, 1961.

[24] D.C. Youla and J.J. Bongiono, "A Feedback Theory of Two- degree-of-Freedom Optimal WienerHopf Design," IEEE Trans. on Automatic Control, vol AC-30, No. 7, pp. 652-665, 1985. 